\title{
Clinical spectrum and genetic landscape for hereditary spastic paraplegias in China
}

En-Lin Dong ${ }^{1 \dagger}$, Chong Wang ${ }^{1 \dagger}$, Shuang $\mathrm{Wu}^{1 \dagger}$, Ying-Qian Lu', Xiao-Hong Lin', Hui-Zhen Su', Miao Zhao ${ }^{1}$, Jin He${ }^{1}$, Li-Xiang $\mathrm{Ma}^{2}$, Ning Wang ${ }^{1,3}$, Wan-Jin Chen ${ }^{1,3^{*}}$ and Xiang Lin ${ }^{1 *}$ (1)

\begin{abstract}
Background: Hereditary spastic paraplegias (HSP) is a heterogeneous group of rare neurodegenerative disorders affecting the corticospinal tracts. To date, more than 78 HSP loci have been mapped to cause HSP. However, both the clinical and mutational spectrum of Chinese patients with HSP remained unclear. In this study, we aim to perform a comprehensive analysis of clinical phenotypes and genetic distributions in a large cohort of Chinese HSP patients, and to elucidate the primary pathogenesis in this population.
\end{abstract}

Methods: We firstly performed next-generation sequencing targeting 149 genes correlated with HSP in 99 index cases of our cohort. Multiplex ligation-dependent probe amplification testing was further carried out among those patients without known disease-causing gene mutations. We simultaneously performed a retrospective study on the reported patients exhibiting HSP in other Chinese cohorts. All clinical and molecular characterization from above two groups of Chinese HSP patients were analyzed and summarized. Eventually, we further validated the cellular changes in fibroblasts of two major spastic paraplegia (SPG) patients (SPG4 and SPG11) in vitro.

Results: Most patients of ADHSP (94\%) are pure forms, whereas most patients of ARHSP (78\%) tend to be complicated forms. In ADHSP, we found that SPG4 (79\%) was the most prevalent, followed by SPG3A (11\%), SPG6 (4\%) and SPG33 (2\%). Subtle mutations were the common genetic cause for SPG4 patients and most of them located in AAA cassette domain of spastin protein. In ARHSP, the most common subtype was SPG11 (53\%), followed by SPG5 (32\%), SPG35 (6\%) and SPG46 (3\%). Moreover, haplotype analysis showed a unique haplotype was shared in 14 families carrying c.334C > T (p.R112*) mutation in CYP7B1 gene, suggesting the founder effect. Functionally, we observed significantly different patterns of mitochondrial dynamics and network, decreased mitochondrial membrane potential $(\Delta \psi \mathrm{m})$, increased reactive oxygen species and reduced ATP content in SPG4 fibroblasts. Moreover, we also found the enlargement of LAMP1-positive organelles and abnormal accumulation of autolysosomes in SPG11 fibroblasts.

Conclusions: Our study present a comprehensive clinical spectrum and genetic landscape for HSP in China. We have also provided additional evidences for mitochondrial and autolysosomal-mediated pathways in the pathogenesis of HSP.

Keywords: Hereditary spastic paraplegias, Clinical features, Mutational spectrum, Heterogeneity, Founder effect, Mitochondria, Autolysosomes

\footnotetext{
* Correspondence: wanjinchen75@fjmu.edu.cn; linxiang1988@fjmu.edu.cn

†En-Lin Dong, Chong Wang and Shuang Wu contributed equally to this work.

${ }^{1}$ Department of Neurology and Institute of Neurology, The First Affiliated Hospital of Fujian Medical University, Fuzhou 350005, China

Full list of author information is available at the end of the article
}

(c) The Author(s). 2018 Open Access This article is distributed under the terms of the Creative Commons Attribution 4.0 International License (http://creativecommons.org/licenses/by/4.0/), which permits unrestricted use, distribution, and reproduction in any medium, provided you give appropriate credit to the original author(s) and the source, provide a link to the Creative Commons license, and indicate if changes were made. The Creative Commons Public Domain Dedication waiver (http://creativecommons.org/publicdomain/zero/1.0/) applies to the data made available in this article, unless otherwise stated. 


\section{Background}

Hereditary spastic paraplegias (HSP) refer to a heterogeneous group of rare neurodegenerative disorders. HSP have a variable age at onset (AAO) and are mainly characterized by slow progressive bilateral spasticity and weakness of lower limbs [1]. The estimated prevalence of HSP is about 2-10/100000 in the general population [2]. Clinically, HSP cases are divided into pure and complicated forms. Pure form is characterized by pyramidal signs, associated with isolated spasticity and weakness confined to the lower limbs [3]. These patients rarely need wheel chairs but may use crutches during the disease course, even they usually have normal lifespan [4]. However, additional severe neurological symptoms can be exhibited in the complicated form, such as ataxia, dysarthria, cognitive impairment, mental retardation, epilepsy, peripheral neuropathy, as well as extra-neurological signs like ophthalmoplegia [5]. The functional handicap and lifespan are associated with the full clinical manifestation in complicated form [6].

Genetically, HSP are highly heterogeneous, with 78 spastic paraplegia (SPG) associated loci and more than 60 identified genes [5, 7]. Moreover, at least 20 additional genes have recently been correlated to HSP $[5,6]$. HSP can be transmitted with all classical modes of inheritance, including autosomal dominant (AD), autosomal recessive (AR), X-linked or mitochondrial maternal transmission. Autosomal dominant hereditary spastic paraplegia (ADHSP) is the most prevalent form of HSP and accounts for approximately $70 \%$ of cases [8]. SPG4 (50\%), SPG3A (10\%), SPG31 (4.5\%) and SPG10 (2.5\%) are the most common causes of ADHSP, in decreasing order of prevalence [6]. Autosomal recessive HSP (ARHSP) is the next largest group of HSP. Mutations in the CYP7B1 (SPG5), SPG7 (SPG7), SPG11 (SPG11) and ZFYVE26 (SPG15) gene are described as the most frequent causes for ARHSP $[9,10]$. However, their relative frequencies vary largely depending on the distinct geographical origin.

Membrane trafficking and axonal transport are emerging as potentially main scenario for HSP [4]. The considerable known HSP causative genes encode proteins mainly involved in microtubule dynamics, endoplasmic reticulum morphogenesis, vesicular formation, lipid metabolism and endosomal functions [3, 11]. However, growing evidences revealed that above theories cannot fully explain the etiology of HSP [12]. Identifying the role of responsible genes could have far-reaching effects on patients, as it would reveal the cause of the disease, and help to better understand the correlation between genotypes and phenotypes. With the growing number of causative genes and the complexity of genotypes and phenotypes, it is difficult to achieve a definite genetic diagnosis in many affected individuals using traditional sanger sequencing. To date, comprehensive review of clinical spectrum and genetic landscape in a large cohort of HSP patients have not yet been presented in China.

In the present study, we firstly investigated the causative genes in 99 both ADHSP and ARHSP families in our cohort using the targeted next generation sequencing (NGS) or multiplex ligation-dependent probe amplification (MLPA) approach. We then retrospectively analyzed and summarized the clinical spectrum and genetic landscape in 47 documented families of our cohort, and in 140 confirmed HSP families of other reported cohorts in China. During the course of these studies, we discovered that SPG4 (79\%), SPG3A (11\%), SPG6 (4\%) and SPG33 (2\%) were the most frequently found in Chinese ADHSP patients, and were mainly pure forms with wide range AAO. Meanwhile, Chinese ARHSP patients were more complex in clinical terms with early AAO. The major subtypes of ARHSP in decrease order of frequency were SPG11 (53\%), SPG5 (32\%), SPG35 (6\%) and SPG46 (3\%). Moreover, we determined that the high prevalence of SPG5 resulted from the founder effect. On these basis, we further conducted functional studies on two major subtypes, SPG4 and SPG11, patients derived fibroblasts to elucidate the pathogenic mechanisms. Overall, these results have provided a new perspective to assess the clinical features, genetic distribution and physiopathology for Chinses HSP patients.

\section{Methods}

\section{Subjects recruitment}

Subjects participated in our cohort were recruited from the Department of Neurology, First Affiliated Hospital of Fujian Medical University. In total, 99 families fulfilling the clinical diagnostic criteria for HSP were enrolled irrespective of their genetic diagnosis [13]. Patients in other cohort were collected by review of 41 reported studies exhibiting HSP in China (Additional file 1: Table S1). A total of 418 HSP patients from 140 families were included.

In addition, 200 unrelated Chinese individuals without history of spastic paraplegia were selected as the control group.

\section{Targeted NGS and data analysis}

Genomic DNA was extracted from peripheral vein blood using a QIAamp DNA Blood Mini Kit (Qiagen, Hilden, Germany) according to the manufacturer's instructions. Targeted NGS panel was designed to cover 149 genes known to be correlated with HSP (Additional file 1: Table S2). The whole exons, along with 20 base pairs of flanking sequences, of targeted genes were enriched with the TruSeq DNA LT Sample Prep Kit v2 (Illumina) according to the manufacturer's standard protocol. DNA libraries were 
subjected to paired-end sequencing on the Illumina High Seq 3000 platform. After eliminating the low-quality FASTQ sequencing reads by Trim Galore, we mapped the remaining high-quality reads to the human reference genome (UCSC hg19) with Burrows-Wheeler Aligner. The variants were called using Genome Analysis Tool Kit, and were annotated with the annotate variation.

All the variants were further filtered by $1000 \mathrm{Ge}$ nomes Project (http://phase3browser.1000genomes.org/index.html), Exome Sequencing Project (http:// evs.gs.washington.edu/EVS/) and Exome Aggregation Consortium database (http://exac.broadinstitute.org/). The property of variants, either benign or pathogenic, were further analyzed by SIFT (http://sift.jcvi.org), PolyPhen-2 (http://genetics.bwh.harvard.edu/pph2/), and Mutation Taster (http://www.mutationtaster.org). Finally, the pathogenic mutations were compared with the Human Gene Mutation Database (http:// www.hgmd.cf.ac.uk/) to determine whether they were known or novel mutations.

\section{Sanger sequencing}

Specific primers for amplification of mutations were designed by the Primer 5 software listed in Additional file 1: Table S3. The targeted regions were amplified with polymerase chain reaction (PCR) in a 2720 Thermal Cycler (ABI). After purification, the amplified products were electrophoresed on an ABI 3730XL Automated DNA analyser (PE Applied Biosystems, Foster City, CA) according to standard protocols. The sequencing results were assembled with chromas software.

\section{MLPA testing}

MLPA testing was performed in the patients without known disease-causing gene mutations for detection of large deletions or copy number variations. The MLPA analyses were performed according to the manufacturer's protocol using the following SALSA MLPA probemixes: P165-C2 (coverage: ATL1, SPAST), P211-B4 (coverage: SPAST, NIPA1), P306-B1 (coverage: SPG11) and P213-B2 (coverage: REEP1, SPG7) (MRC-Holland, Amsterdam, the Netherlands). Data were collected by Genemapper 3.0 (Applied Biosystems, California, USA) and analyzed using Coffalyser software.

\section{Haplotype analysis}

Based on the database of Southern Han Chinese in Haplotype Map Project (https://www.ncbi.nlm.nih.gov/variation/tools/1000genomes/), 9 tagSNPs (rs9298109, rs6985116, rs7842714, rs116843046, rs200737038, rs4465006, rs3779869, rs8192906 and rs4367588) were selected for further haplotype analysis of unrelated SPG5 families. These tagSNPs are linkage to CYP7B1 gene and spanning $\sim 1.5-\mathrm{Mb}$ interval. PCR and sanger sequencing were used to identify the specific genotype of tagSNP. Primers for amplification were listed in Additional file 1: Table S3.

\section{Study on the mutational function in vitro DNA constructs and cell transfection}

The human M1- and M87-spastin cDNAs was prepared as described previously [14]. Briefly, wild-type spastin cDNAs was cloned into the BamHI and NotI site of the eukaryotic expression vector of pCMV6-Entry, with $\mathrm{HA}$ tag at the $\mathrm{N}$ terminus. To generate mutagenesis plasmid, the Mut Express II Fast Mutagenesis Kit V2 (Vazyme) was used according to the manufacturer's instructions. The construction was verified by DNA sequencing after ligation, transformation and extraction. The primers for plasmid construct were summarized in Additional file 1: Table S3.

HEK293T cells were cultured in Dulbecco's modified Eagle's medium (DMEM) supplemented with $10 \%$ fetal bovine serum (FBS) and maintained at $37{ }^{\circ} \mathrm{C}$ under $5 \%$ $\mathrm{CO}_{2}$ /air. Transient transfections were performed using Lipofectamine 3000 (Thermo Scientific) for plasmid DNAs.

\section{Primary fibroblasts culture and treatment}

Primary fibroblast cell lines were established from skin biopsies with punches (Electron microscopy sciences), obtained from the probands of family F12 of SPG4 and family F4 of SPG11. Fibroblasts were maintained in DMEM supplemented with 20\% FBS and 1\% penicillin/ streptomycin.

Autophagy was induced by amino acid and serum starvation in Earle's Balanced Salt Solution (Thermo Scientific) for $6 \mathrm{~h}$.

\section{Reverse transcription polymerase chain reaction (RT-PCR) and quantification PCR (qPCR)}

Total RNA from leukocytes or fibroblasts were extracted with the TRIzol kit (Invitrogen) according to the manufacturer's instructions. A $0.5-\mu \mathrm{g}$ aliquot of total RNA was reversely transcribed into cDNA.

RT-PCR was performed in a 2720 Thermal Cycler (ABI), and the resulting products were visualized on 2.5\% agarose gels under UV transilluminator (Tanon, China). QPCR were performed with SYBR@Premix EX TaqTM II kit (Takara) and run on an Applied Biosystems Stepone Real-time PCR System. The primers for amplification are listed in Additional file 1: Table S3. 


\section{Detection of mitochondrial membrane potential (MMP, $\Delta \psi m)$ by JC- 1}

Fibroblast cells were cultured on glass slide. JC-1 dye solution was added to cells in medium and incubated for $20 \mathrm{~min}$ at $37^{\circ} \mathrm{C}$ (Addition file 1: Table S4), according to the manufacture's recommended protocol. After washing with cold incubation buffer solution, JC-1 staining of mitochondria in live cells was immediately observed using a Leica TCS SP8 confocal system. The wavelength at excitation/emission $525 / 590 \mathrm{~nm}$ was used to assess JC-1 aggregates, and at excitation/emission $488 / 525 \mathrm{~nm}$ was used to assess JC-1 monomer.

\section{Detection of intracellular reactive oxygen species level by DCFH-DA}

Reactive oxygen species (ROS) in fibroblasts were detected using a fluorescent probe, $2^{\prime} 7^{\prime}$-dichlorofluorescein diacetate (DCFH-DA). DCFH-DA is hydrolyzed to DCFH, which further be oxidized to fluorescent DCF by intracellular ROS. Briefly, fibroblasts seeded on glass slide were rinsed with $1 \times \mathrm{PBS}$ and incubated with DCFH-DA for $20 \mathrm{~min}$ at $37^{\circ} \mathrm{C}$ (Addition file 1: Table S4), according to the manufacture's recommended protocol. After washing with $1 \times$ PBS, DCFH-DA labeled mitochondria in live cells were immediately observed using a Leica TCS SP8 confocal system. The wavelength at $488 \mathrm{~nm}$ excitation and $525 \mathrm{~nm}$ emission was used to assess DCF.

\section{Measurement of ATP content}

ATP content was determined in fibroblasts cell lysates using an ATP bioluminescence assay kit (Addition file 1: Table S4), according to the manufacture's recommended protocol. Measurements were performed in a luminometer (Tecan, Austria). The relative ATP content was recorded as ATP value $(\mathrm{nmol}) /$ protein value $(\mathrm{mg})$ ratio.

\section{Immunocytochemistry (ICH) and quantification}

The fibroblasts were fixed with $4 \%$ paraformaldehyde (10-20 $\mathrm{min}$ at room temperature), rinsed three times with $1 \times$ PBS (Medicago AB) and incubated in a blocking buffer $(10 \%$ donkey serum and $0.2 \%$ triton $\mathrm{X}-100$ in PBS) for $1 \mathrm{~h}$ and then incubated with primary antibodies (Additional file 1: Table S4) overnight in a refrigerator at $4{ }^{\circ} \mathrm{C}$. Fluorescence conjugated secondary antibodies (Thermo Scientific) and DAPI (Sigma) were used at 1:1000 dilution. Images were captured by a Leica TCS SP8 confocal system.

Image-J software (NIH, MD, USA) was used for further quantification of the cell population, particle number, lysosome diameter and fluorescence intensity. Quantification was performed by a person blind to the experiment and replicated in four random visual fields from three independent experiments.

\section{Western blotting}

The cells were harvested in the cell lysis reagent (Sigma) supplemented with a $1 \%$ protease inhibitor PMSF (Beyotime) and centrifuged at 13,000 rpm for $20 \mathrm{~min}$ at $4{ }^{\circ} \mathrm{C}$. Protein extracts were separated on $12 \%$ SDS-PAGE gels and immunoblotted with primary antibodies listed in Additional file 1: Table S4. HRP-conjugated secondary antibodies (1:5000, Santa Cruz) were used to detect primary antibodies and proteins were visualized by chemiluminescence (Millipore).

\section{Statistical analysis}

All data were presented as the mean \pm SEM. SPSS 16.0 was used for statistical comparisons, and significance was determined using the Student's $t$-test. Differences were considered statistically significant when the $P$ value was less than $0.05\left({ }^{\prime \prime}\right)$.

\section{Results}

NGS targeting 149 genes correlated with HSP was performed in all probands of our cohort. In aggregate, approximately $99.9 \%$ target bases were covered by the probe and over $82.3 \%$ of bases were covered at greater than $50 \times$, suggesting an accepted threshold for variant calling. After filtering the sequence data down to nonsynonymous or loss-of-function (LOF) variants with allele frequencies less than $1 \%$ in the 1000 Genomes, ESP5400 and ExAC, we finally identified 37 novel or known pathogenic variants in both 22 ADHSP and 25 ARHSP families. All these mutations have been confirmed by sanger sequencing and were not detected in 200 ethnically matched controls. Moreover, exon deletion of SPAST gene was detected among three SPG4 families by MLPA. With retrospective analysis, 89 ADHSP and 51 ARHSP families in other Chinese cohorts were also included in the present study (Additional file 1: Table S1).

Collectively, a considerable number of ADHSP patients (94\%) were pure forms, whereas most ARHSP patients (78\%) tended to be complicated forms (Fig. 1a and b). In ADHSP, we found that SPG4 was the most frequent subtype, account for $79 \%$ of all cases with causal dominant genes in China (Fig. 1c). SPG4 was followed by SPG3A (11\%), SPG6 (4\%) and SPG33 (2\%). In ARHSP, the most common subtype was SPG11 (53\%), followed by SPG5 (32\%), SPG35 (6\%) and SPG46 (3\%) (Fig. 1d). Importantly, some rare subtypes of our cohort patients including SPG8 (1\%), SPG28 (1\%), SPG30 (1\%), SPG43 (1\%) and SPG54 (1\%) were firstly presented in China. 


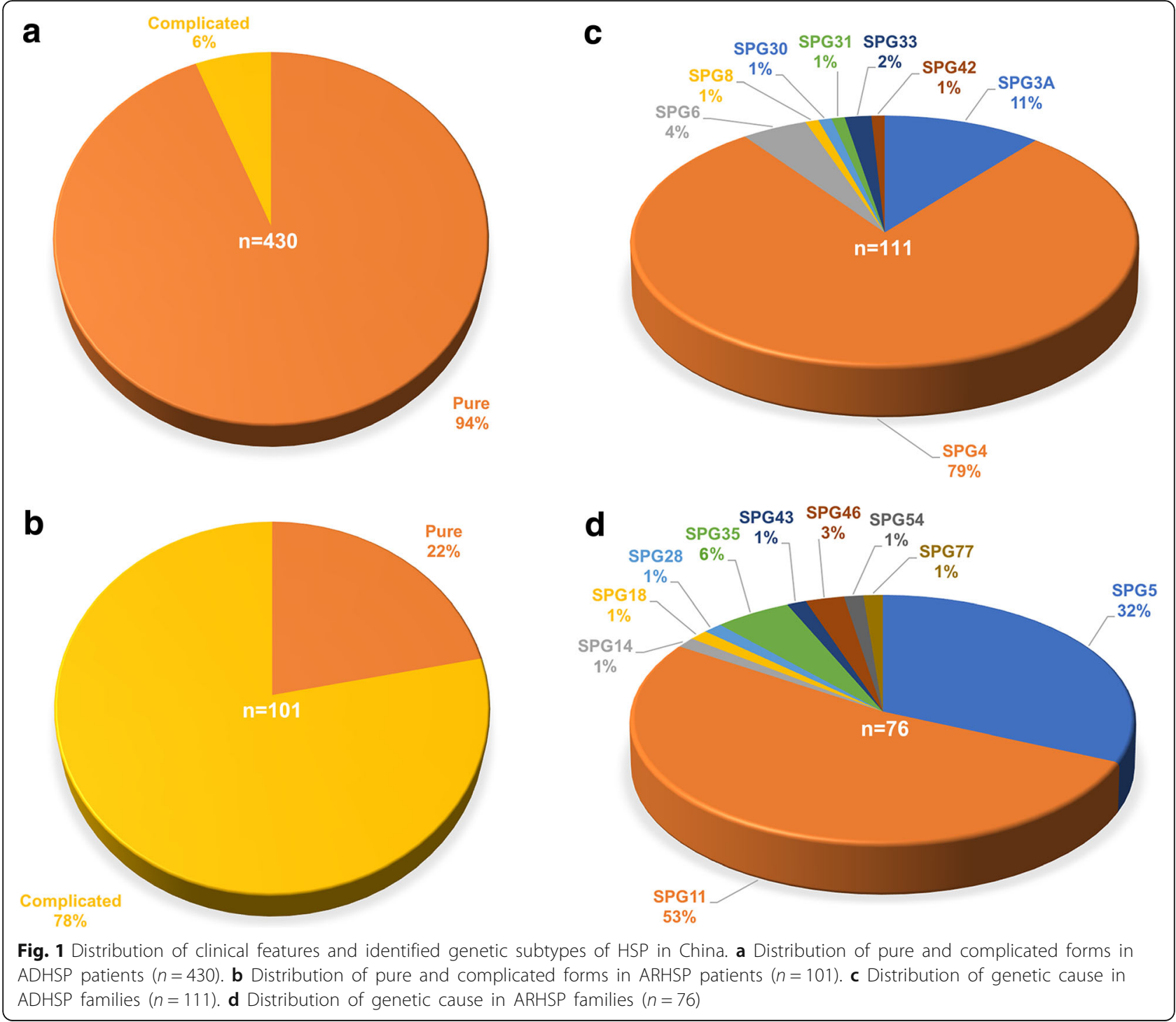

The clinical features and mutational spectrum in Chinese ADHSP patients

\section{Mutations in SPAST gene cause ADHSP}

SPG4 is caused by mutations in SPAST gene [15]. The clinical features of Chinese SPG4 patients were summarized in Additional file 1: Table S5. The mean $\pm \mathrm{SD}$ AAO in these patients was $25.01 \pm 15.84$ years, ranging from 1 to 62 years old. The mean \pm SD disease duration was $17.91 \pm 14.98$ years. Most cases (93.8\%) had the core features of HSP, and were considered as prototypical pure forms. Moreover, peripheral neuropathy was the most common symptom in complicated patients.

Spastin, encoded by SPAST gene, contains four functional domains including transmembrane (TM) domain, microtubule interacting and trafficking (MIT) domain, microtubule-binding domain (MTBD), and
AAA ( $A$ TPases associated with diverse cellular activities) cassette domain [16]. To date, 46 subtle mutations in SPAST gene have been reported in Chinese SPG4 patients (Additional file 1: Table S1). In our cohort, 16 SPG4 families were identified to carry 14 pathogenetic mutations: seven were missense mutations, two were frameshift mutations, one was stop-gain mutation, two were splicing mutations and two were exons deletion (Additional file 2: Figure S1). All of these 58 subtle mutations widely distributed in four domains of spastin protein and mainly located in the AAA cassette (Fig. 2a and b), accounting for up to $78.3 \%$. Except for subtle mutations, 16 kinds of large deletions or duplications of SPAST gene have also been detected, of which the exon 11-12 deletion was the most common mode (Fig. 2c). By comparing the frequency, we further discovered that subtle 


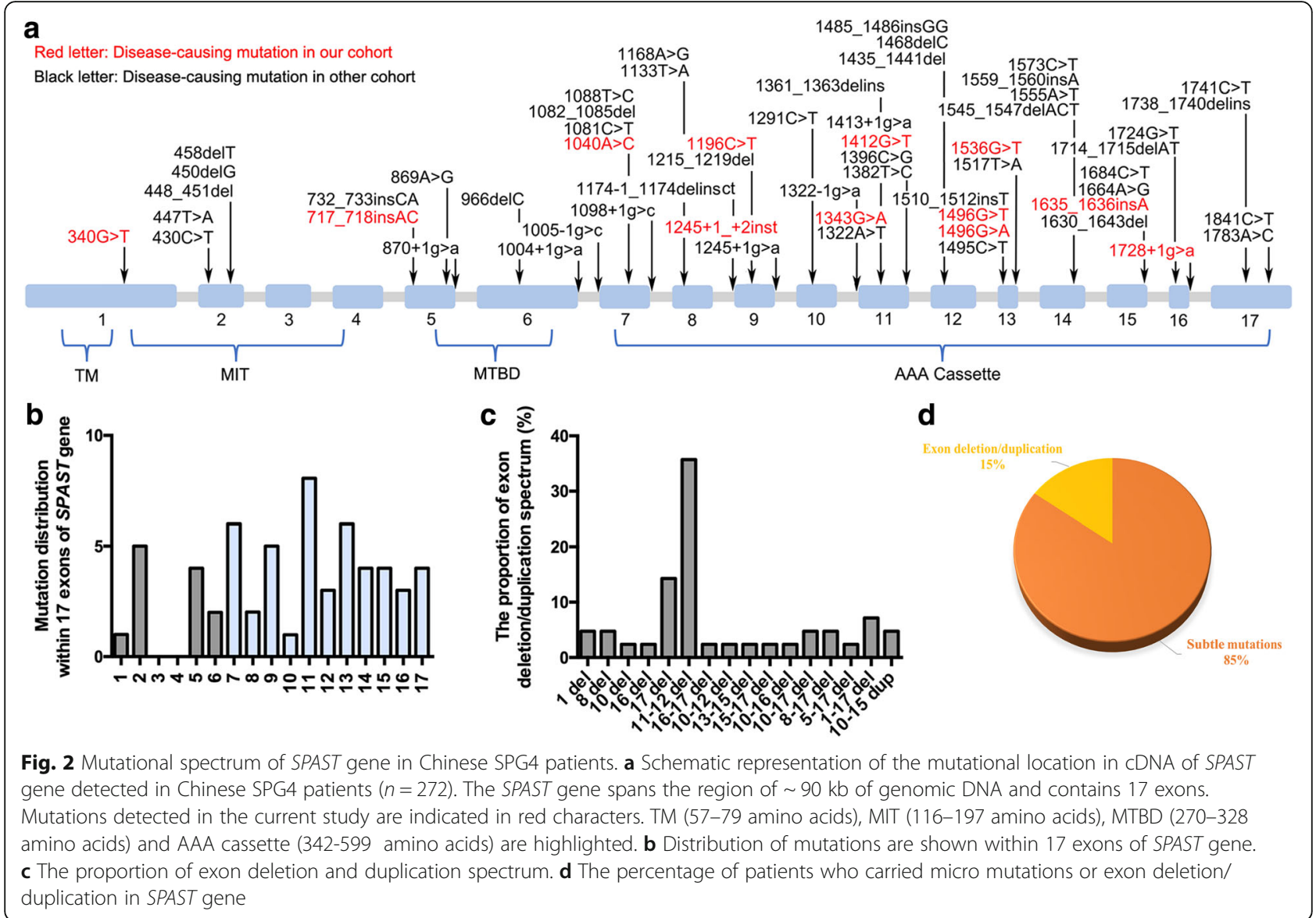

mutations were the much more common (85\%) genetic cause for SPG4 (Fig. 2d).

Four novel mutations, c.717_718insAC (p.S243Lfs"12), c.1040A > C (p.Q347P), c.1245 + 1_c.1245 + 2insT and c.1635_1636insA (p.G546Rfs"3) were identified in our cohort. Based on the standards and guidelines for the interpretation of sequence variants [17], p.S243Lfs" 12 and p.G546Rfs" 3 were considered as both LOF mutations (Additional file 2: Figure S2), which was the very strong evidence for pathogenic effect. To further confirm the pathogenesis of c.1040A >C and c.1245+1_c.1245+ 2insT, we studied the functional effect on spastin. As a result of two different initiation codons, two primary isoforms of spastin were synthesized: a full-length isoform called M1 and a slightly shorter isoform called M87. M87 was detectably presented in all tissues, whereas M1 was only expressed in adult spinal cord [12]. Therefore, we introduced the p.Q347P missense mutation into M1- and M87-spastin expression HA-tag vectors and overexpressed them in HEK $293 \mathrm{~T}$ cell (Fig. 3a). Immunoblotting of HA showed that spastin level of p.Q347P was found to be comparable to that of WT (Additional file 2: Figure S2). To further study its influence on stability of spastin, we performed cycloheximide (CHX, $40 \mu \mathrm{g} / \mathrm{ml})$ chase assay to inhibit de-novo protein synthesis and reassessed the protein expression levels after different treatment durations. The treatment of CHX resulted in rapid degradation of p.Q347P protein (Fig. 3b and c; Additional file 2: Figure $\mathrm{S} 2$ ), indicating that the missense mutation affected protein half-life of spastin. In addition, we also validated the effect of splicing mutation c. $1245+1$ _c. $1245+2$ insT on patient's leukocytes derived mRNA. A different product in size from the RT-PCR of SPAST cDNA from the patient was detected (Fig. 3d), indicating the presence of an aberrant splice event (Fig. 3e). The sequencing of amplification products confirmed that alternative splicing result in the skipping of exon 9 in mutant transcript (Fig. 3f).

\section{Mutations in ATL1, KIAA0196, KIF1A and ZFYVE27 gene cause ADHSP}

Mutations in KIAA0196 (SPG8), KIF1A (SPG30) and ZFYVE27 (SPG33) gene of our cohort ADHSP patients were firstly presented in China. Three missense mutations and one frameshift mutation of these genes (p.T845A in KIAA0196, p.I37T in KIF1A, p. N88Rfs" 7 and p.A290T in ZFYVE27) were detected in four ADHSP families 


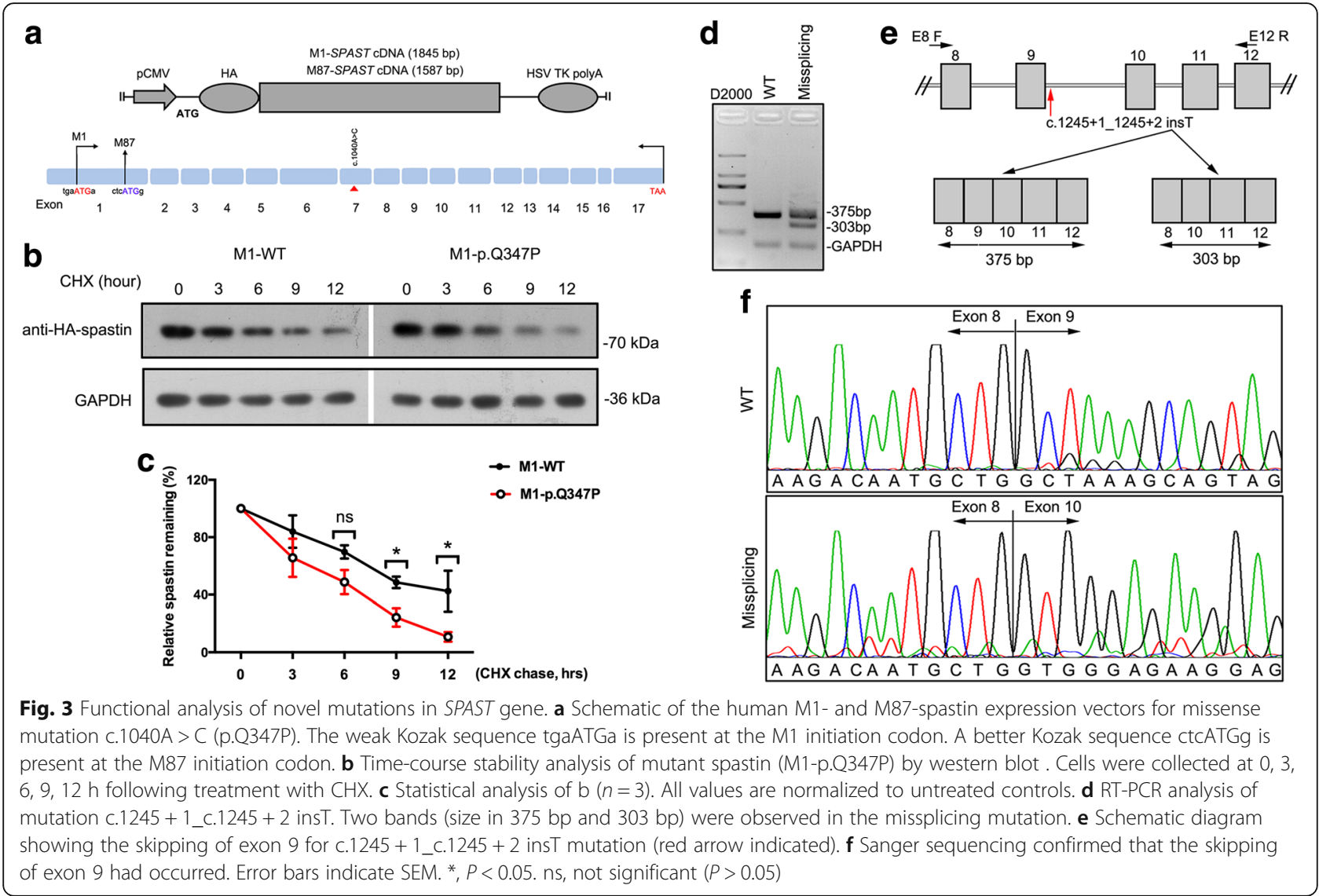

(Additional file 2: Figure S3). Patients carrying p.T845A in KIAA0196 and p.A290T in ZFYVE27, were classified as complicated HSP, whereas others presented as pure form. Structural magnetic resonance images (MRI) of the complicated patient carrying p.A290T revealed agenesis of corpus callosum and leukoencephalopathy. In addition, we also identified two missense mutations (p.A350S and p.R416C) of ATL1 (SPG3A) in one family with pure form and another family with complicated form, respectively (Additional file 2: Figure S3). Both probands with ATL1 mutations had a very young AAO. Intriguingly, the mother of the proband carrying the reported mutation p.R416C, remained asymptomatic presently.

\section{The clinical features and mutational spectrum in Chinese ARHSP \\ Mutations in SPG11 gene cause ARHSP}

SPG11 is caused by mutations in SPG11 gene [18]. The clinical characterization of Chinese SPG11 patients were summarized in Additional file 1: Table S6. In general, SPG11 patients had early AAO $(12.71 \pm 4.48$ years, mean \pm SD), ranging from 4 to 20 years old. The mean \pm SD disease duration was $12.46 \pm 8.43$ years. The majority of patients (78.5\%) initially presented with gait disturbance. Except for the typical manifestation of HSP, most cases combined with cognitive deficit (78.9\%), dysarthria (73.1\%), mental retardation (53.9\%) and peripheral neuropathy (61.6\%). Notably, $92.3 \%$ of SPG11 patients presented a typical sign of thin corpus callosum on MRI.

A total of 31 subtle mutations have been detected in Chinese SPG11 patients so far (Fig. 4a). In our cohort, we identified five LOF mutations (p.M245Vfs"2, p.I415", p.M1272Ifs" 6, p.R2031" and p.W2234") of SPG11 gene in 4 SPG11 families (Fig. 4b). Sanger sequencing of these mutations in other family members showed a co-segregation of genotype with the disease phenotype. However, we did not find obvious clustering of these 36 mutations on the functional domain of spatacsin protein, encoded by SPG11 gene (Fig. 4a).

\section{Mutations in CYP7B1 gene cause ARHSP}

SPG5 is caused by mutations in CYP7B1 gene [19]. The mean \pm SD AAO in Chinese SPG5 patients was $16.88 \pm$ 10.39 years, ranging from 3 to 44 years old, with a peak in the first two decades. The mean \pm SD disease duration was $9.93 \pm 7.17$ years. More than half of the patients (57.1\%) presented as complicated forms. Interestingly, 


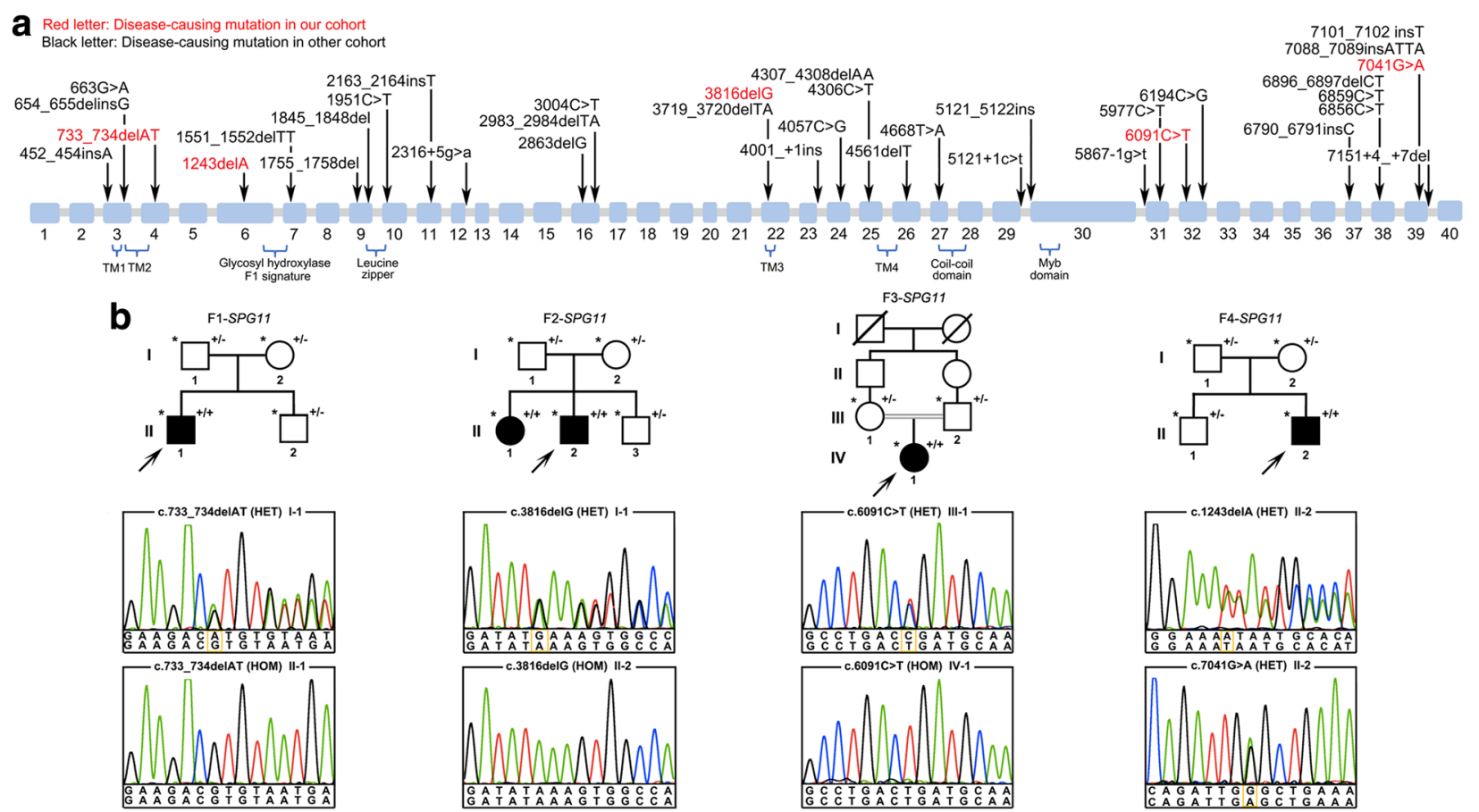

Fig. 4 Mutational spectrum of SPG11 gene in Chinese SPG11 patients. a Schematic representation of the mutational location in CDNA of SPG11 gene detected in Chinese SPG11 patients $(n=52)$. Mutations detected in our cohort are indicated in red characters. Four transmembrane domains (TM1, TM2, TM3 and TM4), Glycosyl hydroxylase F1 signature, Leucine zipper, Coil-coil domain and Myb domain are highlighted. b Pedigree chromatograms and sanger sequencing of four SPG11 families in our cohort. Three probands carried homozygous LOF mutations: c.733_734delAT (p.M245Vfs ${ }^{*}$ ) in family F1, c.3816 delG (p.M1272lfs ${ }^{*}$ ) in family F2 and c.6091C > T (p.R2031 ${ }^{*}$ ) in family F3. The index case in family F4 carried compound heterozygous LOF mutations: c.1243delA (p.1415*) (I-2) and c 7041G (p.W2234") (I-1). Symbol with "+/+" indicate patient. Symbol with " $+/{ }^{*}$ " indicate mutation carrier; Symbol with "**" indicate the member whose sample was available. Mutations were marked by yellow box

white matter lesions were observed in $50 \%$ of complicated SPG5 patients (Additional file 1: Table S7).

In our cohort, mutations in CYP7B1 gene was identified in 16 SPG5 kindred (Additional file 2: Figure S4). Among these, 14 families carried the known nonsense mutation c.334 C > T (p.R112") [20], which was homozygous in 13 families, accounting for up to $92.9 \%$. Haplotype analysis was further performed on members of the 16 unrelated families, and revealed that cases with the mutation c.334C > T (p.R112") shared the same unique haplotype in the $1.5-\mathrm{Mb}$ interval between tagSNP rs9298109 and rs4367588 (Additional file 1: Table S8). This result implied that all the specific SPG5 patients carrying the homozygous mutation c.334C > T (p.R112*), originated from the common ancestor, which is termed as 'founder effect' (Additional file 1: Table S8). Besides that, we also identified another four novel mutations (p.L145Cfs"13, p.Y181", p.R370C and p.P397L) of CYP7B1 in another 3 remaining families.

\section{Mutations in DDHD1, FA2H, C19orf12, GBA2, and DDHD2 gene cause ARHSP}

Mutations in DDHD1 (SPG28), C19orf12 (SPG43) and DDHD2 (SPG54) gene of our ARHSP patients were firstly presented in China. One previously mutation (p.G58R in C19orf12) and three novel mutations (p.R740" in DDHD1, p.Y99" and p.R112Q in DDHD2) were identified in three ARHSP families (Additional file 2: Figure S5). All these three families mainly presented with weakness and spasticity restricted to the lower limbs, thus they were considered as a pure form of HSP. In addition, we have also confirmed four novel mutations (p.P148L and p.L131Sfs" 14 in $F A 2 H$ (SPG35), p.W475" and p.R879W in GBA2 (SPG46)) in two ARHSP families (Additional file 2: Figure S5). Both of them showed additional complicated features including dysarthria, cognition impairment, even skin peeling.

\section{Functional characterization of mutant spastin and spatacsin in the patients derived fibroblast cells}

SPG4 and SPG11 are the most common subtypes of ADHSP and ARHSP in China, respectively. The two groups account for up to $68.5 \%$ of all patients. To elucidate their pathogenic mechanism, we further study the function of spastin and spatacsin protein in patients derived fibroblasts. 


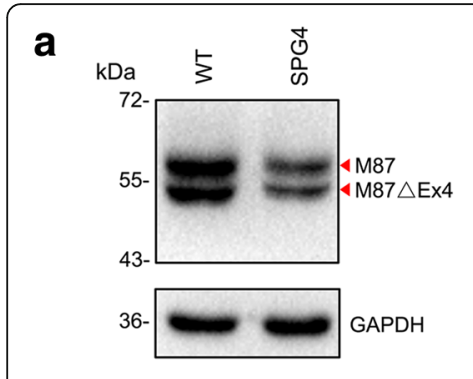

b

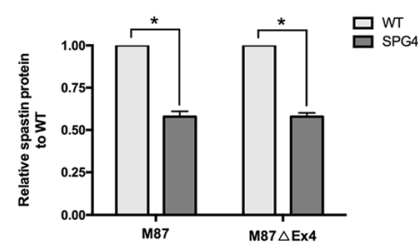

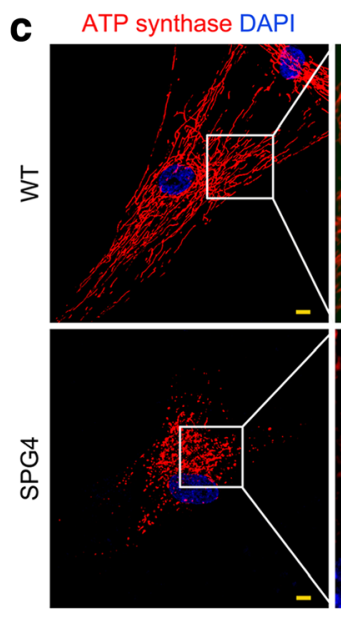

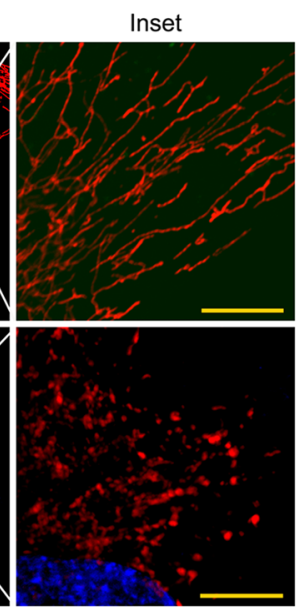

d

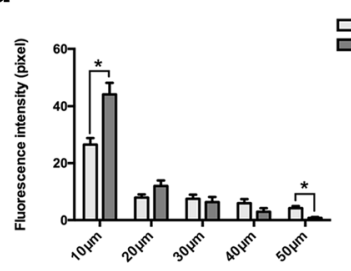

ㅁT
SPG4

.

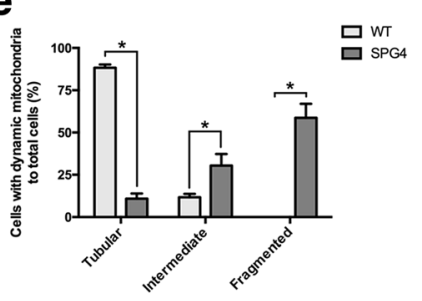

f

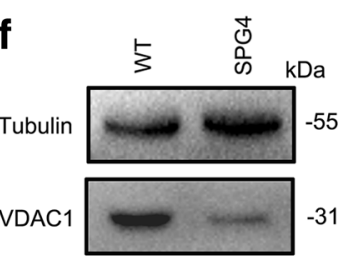

h

g

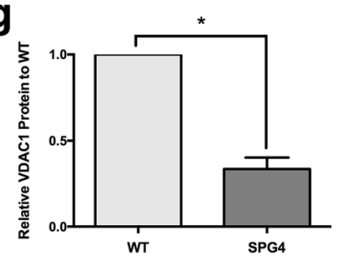

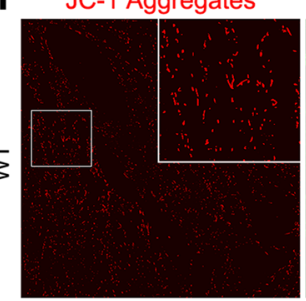

JC-1 Monomer
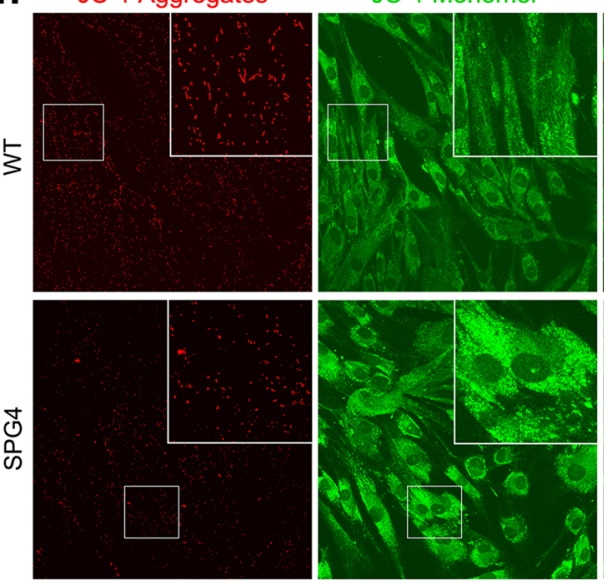
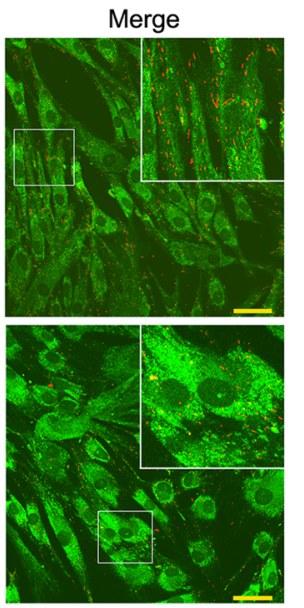
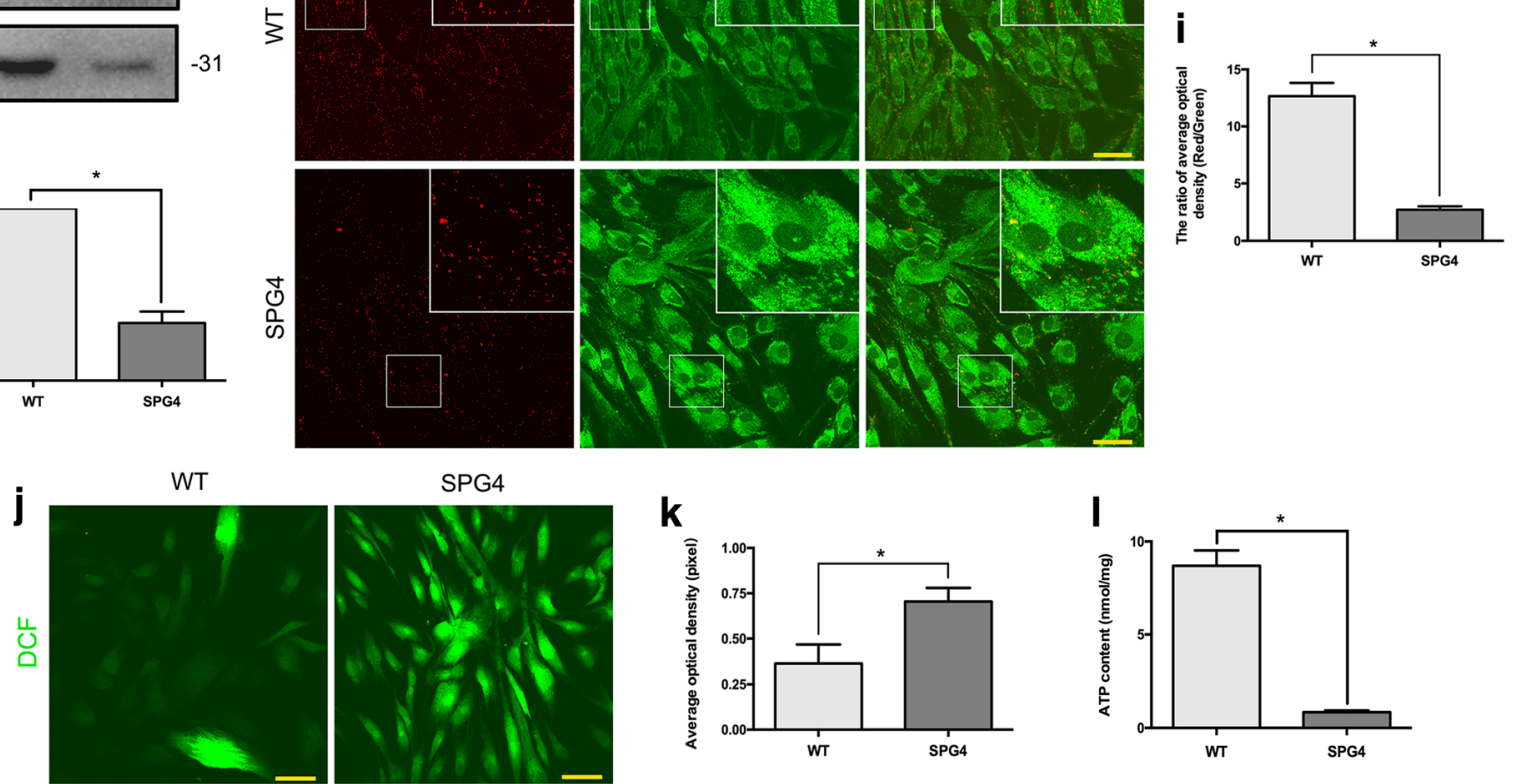

Fig. 5 Mitochondrial dynamics and oxidative metabolism were altered in the SPG4 patient derived fibroblasts. a, b Western blot analysis showed a significant difference in spastin isoforms (M87 and M87 $\triangle$ Ex4) expression levels between the SPG4 patient and WT. c ICH of mitochondria maker (ATP synthase, red) and DAPI (blue) in fibroblasts from the WT (upper panel) and the SPG4 patient (lower panel). Insets in the images are enlarged (original magnification, $\times 8.0)$ to the right. Scale bar, $10 \mu \mathrm{m}$. d Quantification of mitochondria distribution measured from the center of the cells $(n=3,>10$ cells per experiment). The data showed that the number of mitochondria were significantly reduced along the branch in the SPG4 patient derived fibroblasts. e Quantification of cells with different mitochondrial morphology (tubular, intermediate and fragmented patterns) to total cell ( $n=3,>30$ cells per experiment). $\mathbf{f}, \mathbf{g}$ Western blot analysis showed a significant difference in VDAC1 protein expression levels between the SPG4 patient and WT. h JC-1 staining was used to measure MMP $(\Delta \psi \mathrm{m})$ in fibroblasts from the WT (upper panel) and the SPG4 patient (lower panel). Boxed areas are enlarged in the insets. Scale bar, 50 mm. i Statistical analysis of $\mathbf{h}(n=3)$. $\mathbf{j}$ Intracellular ROS visualization using DCFH-DA staining in WT (upper panel) and SPG4 (lower panel). Scale bar, $50 \mu \mathrm{m}$. k Statistical analysis of $\mathbf{j})(n=3)$. I ATP bioluminometers analysis revealed a significant decrease of ATP content in the SPG4 patient. Error bars indicate SEM. *, $P<0.05$

Loss of spastin results in abnormal mitochondrial dynamics and oxidative metabolism

In fact, there are two additional spastin isoforms, $\mathrm{M} 1 \triangle \mathrm{Ex} 4$ and $\mathrm{M} 87 \triangle \mathrm{Ex} 4$, that result from skipping of exon 4. We observed that the M87 and M87 $\triangle \mathrm{Ex} 4$ isoforms were both present in both WT and SPG4 fibroblasts, but not with M1 or M1 $\triangle \mathrm{Ex} 4$ isoforms (Fig. 5a). The level of both two predominant isoforms were 
significantly reduced by $43 \%$ in SPG4 ( $P<0.001$, Fig. 5 b). The premature termination codon within the AAA cassette domain of spastin is expected to induce nonsense mediated mRNA decay [21]. Consistent with this, we did not find the truncated isoforms $(\sim 50 \mathrm{kDa})$ in the SPG4 fibroblasts (Fig. 5a).

The spastin expression levels are the limiting factor in microtubule severing to maintain microtubule network. Mitochondrial motility and regional distribution have been reported to closely interact with cytoskeletal tracks, such as microtubules and actin filaments [22]. To evaluate the mitochondrial distribution pattern in SPG4, mitochondria were visualized using antibody against ATP synthase. That we observed a more pronounced concentration of mitochondria around the perinuclear region in patient derived fibroblasts $(P<0.001$, Fig. $5 c$ and d), suggesting the intracellular trafficking alteration. Intriguingly, we further found more fragmented and unbranched, but fewer tubular mitochondria in SPG4 fibroblasts $(P<0.001$, Fig. $5 c$ and e), which implied the impaired patterns of mitochondrial fission and fusion. Mitochondrial network fragmentation was further assessed by immunoblot analysis of the voltage-dependent anion channel 1(VDAC1), an outer mitochondrial membrane protein, acts as a gatekeeper for the transport of mitochondrial metabolites [23, 24]. Likewise decreased expression of the VDAC1 protein was observed in SPG4 fibroblasts $(\sim 70 \%$ reduction, $P<0.001$, Fig. $6 \mathrm{f}$ and g). We further sought to determine whether the mitochondrial function was altered. Fluorescence indicator, JC-1, was used to validate the level of MMP $(\Delta \psi \mathrm{m})$. We observed the ratio of average optical density (AOD, red to green) was significantly reduced by $80 \%$ in SPG4 $(P<$ 0.001 , Fig. $6 \mathrm{~h}$ and i). The loss of $\Delta \psi \mathrm{m}$ could induce the increase in intracellular ROS [25], which was strongly confirmed by DCFH-DA staining. We found that the ROS level was dramatically increased in SPG4 fibroblasts $(\sim 2$ folds increase on average, $P<0.001$, Fig. $6 j$ and $\mathrm{k}$ ). We further investigated the effect on ATP content. ATP bioluminometers analysis revealed that the relative ATP content was apparently decreased in the SPG4 fibroblasts (mean \pm SEM, WT: $8.70 \pm 0.83 \mathrm{nmol} / \mathrm{mg}$, SPG4: $0.85 \pm 0.09 \mathrm{nmol} /$ $\mathrm{mg}, P<0.001$, Fig. 6l). Thus, the lack of spastin in SPG4 would cause abnormal mitochondrial dynamics and oxidative metabolism.

\section{Loss of spatacsin results in enlarged LAMP1-positive organelles and accumulation of autolysosomes}

QPCR and ICH results showed that spatacsin expression level was significantly decreased by $80 \%$ in SPG11 fibroblasts $(P<0.001$, Fig. $6 a$ and b). Spatacsin has been reported to play a pivotal role in autophagic lysosome reformation, a pathway that generates new lysosomes [26]. With inducing autophagy by starvation of fibroblasts, we found SPG11 fibroblast cells exhibited prominently enlarged organelles immunostained with LAMP1 ( 2.5 folds increase on average, $P<0.001)$, a marker of late endosomes and lysosomes (Fig. 6c and d). Moreover, the number of cells with enlarged LAMP1-positive organelles (LPOs) was also significantly increased in SPG11 ( 8 folds increase on average, $P<$ 0.001 , Fig. 6e), although this had no significant effect on total LAMP1 protein level $(P=0.235$, Fig. $6 f$ and g). Thus, we speculated that depletion of spatacsin would result in the enlarged volume of LPOs, but not in their change of total amount.

Autophagosomes fuse with lysosomes to generate autolysosomes, which is essential for autophay-dependent intracellular clearance. The accumulation of enlarged LPOs reminds us to study any dysfunction in autophagosomes status. Indeed, SPG11 fibroblasts present significantly aggregated mature autophagosomes labeled by p62 $(P<0.001$, Fig. $6 \mathrm{~h}$ and $\mathrm{i})$, and with enhanced p62 protein level $(\sim 2.0$ folds increase on average, $P<0.001$, Fig. $6 \mathrm{j}$ and k). During autophagy, cytosolic LC3 (LC3-I) is continually modified to form a LC3-phosphatidylethanolamine conjugate (LC3-II), which can be recruited to autophagosomal membranes. The ratio of LC3-II to LC3-I is recognized as a marker for monitoring autophagosomes formation [27]. To further validate above finding, we performed immunoblot on LC3 and confirmed a significant increase in the amount of autophagosomes in SPG11 fibroblasts ( 3.0 folds increase on average, $P<0.001$, Fig. $6 \mathrm{l}$ and $\mathrm{m})$. To investigate the spatial relationship between enlarged LPOs and accumulated autophagosomes, fibroblasts were coimmunostained with LC3 and LAMP1. We found that most autophagosomes labeled by LC3 were also positive with the enlarged LPOs labeled by LAMP1 in both groups $(P=0.858$, Fig. $6 n$ and o), indicating that normal fusion of autophagosomes with lysosomes had occurred in spatacsin-deficiency fibroblasts. Overall, the lack of spatacsin in SPG11 would lead to aberrant accumulation of the autolysosomes.

\section{Discussion}

Herein, we have analyzed and summarized 430 of ADHSP patients and 101 of ARHSP patients in China, respectively. In total, 18 SPG subtypes and more than 150 mutations have been uncovered. To the best of our knowledge, this study included the largest HSP cohort in China and presented a comprehensive overview of the clinical spectrum and genetic landscape for HSP.

As in previous studies, AD forms of HSP are mainly pure forms [28]. The AAO had a wide range from infancy to late adulthood $[29,30]$. Mutations in SPAST (SPG4), ATL1 (SPG3A), KIF5A (SPG10) and REEP1 (SPG31) are reported as being responsible for more than $50 \%$ of all cases [6]. The present study has confirmed that SPG4 is the most common subtype of Chinese 


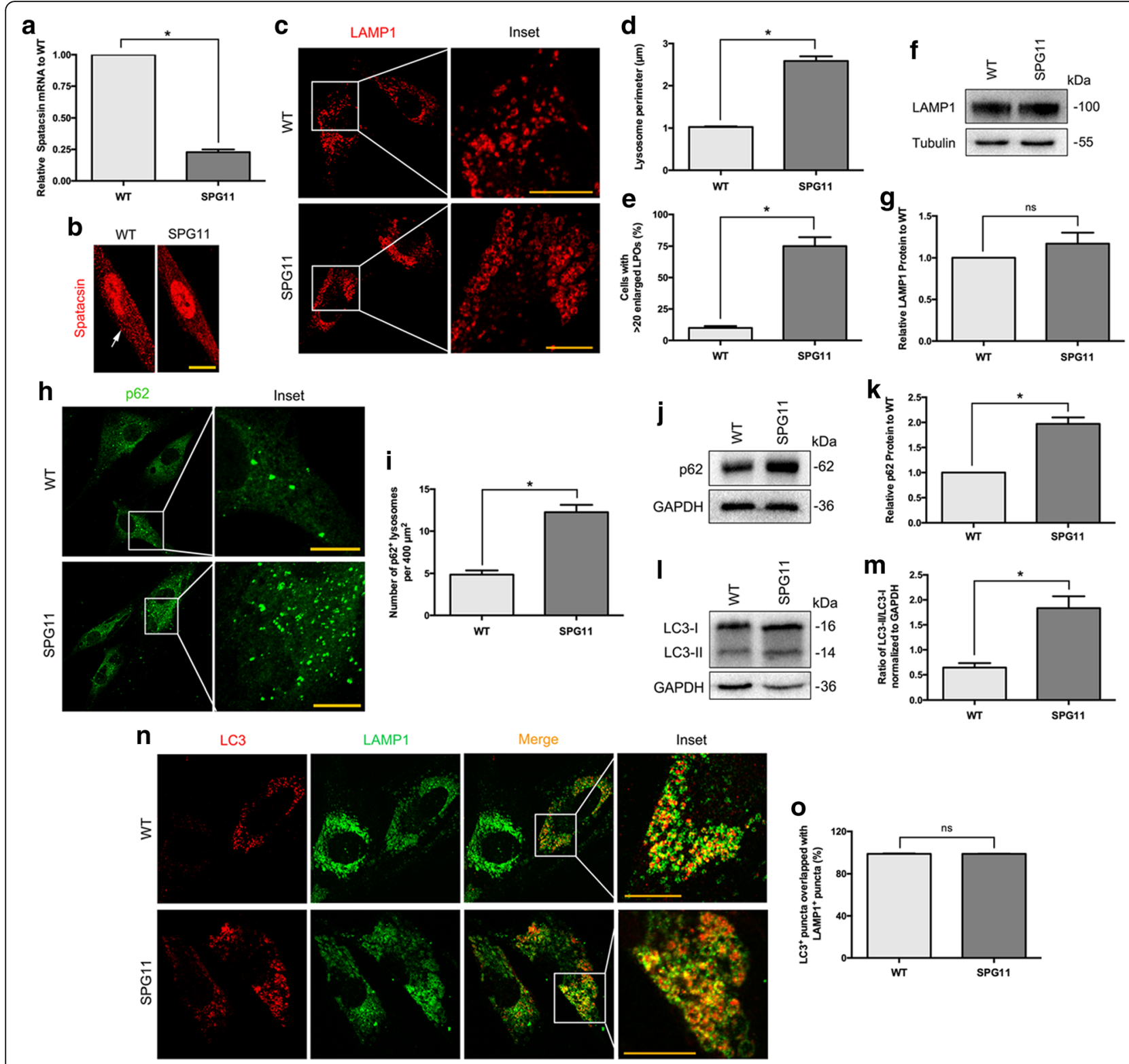

Fig. 6 Fibroblasts from the SPG11 patient exhibit enlarged LAMP1-positive organelles and accumulation of autolysosomes. a QPCR analysis revealed a significantly decreased expression of spatacsin mRNA in the pateint's fibroblasts. $\mathbf{b}$ ICH of spatacsin (red particles, arrow indicated) in fibroblast of WT (left panel) and the SPG4 patient (right panel). c ICH analysis revealed fibroblasts from the SPG11 patient exhibit prominently enlarged LPOs. Insets in the images are enlarged (original magnification, $\times 9.0$ ) to the right. $\mathbf{d}$ Quantification of lysosome perimeter ( 20 cells, $>20$ lysosomes per cell). e Quantification of cells with exceed 20 enlarged LPOs $(n=3,>30$ cells per experiment). $\mathbf{f}, \mathbf{g}$ Western blot analysis showed no significant difference in LAMP1 levels between the SPG11 patient and WT. $\mathbf{h} I \mathrm{CH}$ of p62 (green) in fibroblast of WT (upper panel) and the SPG11 patient (lower panel). Insets in the images are enlarged (original magnification, $\times 9.0$ ) to the right. i Quantification of $\mathrm{h}$ (50 cells). j, k Western blot analysis revealed p62 protein was significantly accumulated in the patient derived fibroblasts. I, $\mathbf{m}$ Western blot analysis showed the ratio of LC3-II/LC3-I was significantly increased in autophagy induced fibroblasts from the SPG11 patient. n, o Co-immunostaining with LC3 (red) and LAMP1 (green), which showed no significant difference of their overlapping between the two groups. Insets in the images are enlarged (original magnification, $\times 10.5)$ to the right. Scale bar, $10 \mu \mathrm{m}$. Error bars indicate SEM. ${ }^{*}, P<0.05$. ns, not significant

ADHSP patients, and followed by SPG3A, SPG6 (NIPA1) and SPG33 (ZFYVE27). Clinically, 94\% of these patients were of pure HSP. However, no mutations were identified in SPG10, which appeared to be a common cause of ADHSP in previous series of other populations [31, 32]. Moreover, SPG8 (KIAA0196), SPG30 (KIF1A), SPG31and SPG42 (SLC33A1) seemed to be quite rare subtypes of Chinese ADHSP patients. 
About $10-40 \%$ of HSP patients harbored subtle mutations and exonic rearrangements in the SPAST gene $[3,33]$. Subtle mutations were confirmed to be the most common genetic cause for Chinese SPG4 patients, and most of them located in AAA casseete domain of Spastin protein. Spastin, a well-known AAA ATPase, is widely expressed in vertebrate cells that sever microtubules [34]. M87 is the much more abundant and effective severing isoform of spastin than M1 [35]. M1 is exclusively presented in adult spinal cord, the location of corticospinal tract axons that degenerate during HSP $[21,35]$. The dynamic microtubule is functionally important for the intracellular organelles trafficking $[14,36]$. Within neurons, only the short microtubules, but not the long ones, are critical for efficient axonal transport [37, 38]. In our cohort, all novel SPAST mutations have been shown to downregulate the expression or stability of M1and M87-spastin protein. Based on SPG4 patient derived fibroblasts, we observed only reduced M87 and $\mathrm{M} 87 \triangle \mathrm{Ex} 4$ isoforms, and that mitochondrial dynamics and network were both altered. Of note, we further found the decreased MMP $(\Delta \psi \mathrm{m})$, increased ROS and reduced ATP content in SPG4 fibroblasts, which have been implicated in cellular injury [39]. The neurite swelling and imbalanced axonal transport have been reported in the SPG4 stem cells derived neurons [21, 40, 41]. Indeed, in postmortem SPG4 patient brain samples, abnormal mitochondria readily accumulated in the cytosol of affected neurons [42]. On these basis, we speculate that the mitochondrial-mediated pathway plays a key role in neuron degeneration in the pathogenesis of SPG4.

In contrary to ADHSP, the AR forms of HSP appear to be more complex in clinical terms, with an early onset of symptoms [6, 43]. SPG11 was the most common cause of ARHSP [44]. In this study, $78 \%$ of Chinese ARHSP patients were suggestive of complicated HSP. Mutations in SPG11 (SPG11) are responsioble for around 53\% of ARHSP cases, and followed by CYP7B1 (SPG5), FA2H (SPG35) and GBA2 (SPG46). However, no mutations were identified in SPG7 (SPG7) and ZFYVE26 (SPG15) gene, which appeared to be a common cause of ARHSP in previous series of other populations $[2,6,9,10,45,46]$. Indeed, subtypes for SPG14, SPG18, SPG28, SPG43, SPG54 and SPG77 of Chinses ARHSP patients are apparently orphan, affecting single family members.

In the present study, the AAO of SPG11 was generally early. The cognitive deficit, dysarthria, mental retardation, thin corpus callosum and axonal peripheral neuropathy were highly suggestive of SPG11. In previous reports, mutations in $C Y P 7 B 1$ were found in both pure and complicated forms of SPG5 [47]. Cerebellar ataxia has been the most common SPG5-related complicated phenotype [48]. Of note, white matter lesions and spastic ataxia seemed to be the two major features in Chinese SPG5 cases, accounting for approximately $50 \%$. It was of interest to note that $82.1 \%$ of these SPG5 patients carried the unique nonsense mutation c.334C $>\mathrm{T}$ (p.R112"). Haplotype analysis revealed that the hotspot mutation is correlated with the founder effect.

Point mutations and rearrangements in SPG11 have been show to make up 20\% of ARHSP [49]. In our cohort, all five mutations of SPG11 were stopgain mutation, with predicting to be LOF. Emerging studies implicated that spastic paraplegia proteins, spatacsin (SPG11) and spastizin (SPG15), were the essential components for the initiation of lysosomal tubulation [26, 50, 51]. Dysfunction of spatacsin leads to impaired axonal maintenance and cargo trafficking in the SPG11 stem cells derived neurons $[52,53]$. Based on SPG11 patient derived fibroblasts, we observed that lack of spatacsin indeed induced the enlargement of LPOs and accumulation of autophagosomes. More importantly, the essence of these abnormally accumulated vesicles was shown to be autolysosomes, whose quantity and quality are crucial for autophagy. Autophagy is an intracellular activity for degradation and recycling of cytoplasmic components to maintain homeostasis [54]. The perturbed autophagy has been linked to several neurodegeneration diseases [55]. Collectively, our findings implied that the presence of autophagic dysfunction is tightly linked to SPG11.

\section{Conclusions}

In summary, our results indicated that subtypes for SPG4, SPG3A, SPG6 and SPG33 were the most frequently found in Chinese ADHSP patients, and were mainly pure forms with wide range AAO. Meanwhile, SPG11 SPG5, SPG35 and SPG46 subtypes were the most frequently found in Chinese ARHSP patients, and were more complex in clinical terms with early AAO. Moreover, we further determined that the high frequency of mutations in CYP7B1 gene resulted from the founder effect. These studies provide the important implications on genetic counseling for suspected HSP cases. All of included patients presented with a wide range of features from asymptom to multi-system involvement, which confirming the marked heterogeneity of HSP. Stressing the mitochondrial and autophagic dysfunction hold promise as a potential therapeutic target to halt the progression of neurodegeneration in HSP. Even though, further study of a large cohort of patients should ascertain the specific correlation of genotype-phenotype in each subtype of HSP. 


\section{Additional files}

Additional file 1: Summary information for mutational spectrum, targeted sequencing genes, amplification primers, antibodies, clinical features and haplotype analysis. Table $\mathbf{S} 1$. A reanalysis of the mutational spectrum in 41 reported studies exhibiting HSP in China. Table S2. HSP related genes included in the targeted sequencing panel. Table S3. Primers for amplification in the experimental procedures. Table S4. List of antibodies, related to immunocytochemistry $(\mathrm{ICH})$ and western blotting (WB) in the experimental procedures. Table S5. Summary of clinical features of SPG4 patients in China ( $n=273$ ). Table S6. Summary of clinical features of SPG11 patients in China ( $n=52)$. Table S7. Summary of clinical features of SPG5 patients in China ( $n=28)$. Table S8. Haplotypes of tagSNPs linkage to CYP7B1 gene in the 16 unrelated SPG5 families. (XLSX 69 kb)

Additional file 2: Pedigrees, sequencing chromatograms of diseasecausing gene related to HSP families in our cohort. Figure S1. Pedigree, sequencing chromatograms of SPAST gene detected in 16 SPG4 families in our cohort. Figure S2. Western blot analysis of novel mutations of SPAST gene in HEK 293T cells. Figure S3. Pedigree, sequencing chromatograms of 6 ADHSP families in our cohort. Figure S4. Pedigree, sequencing chromatograms of CYP7B1 gene detected in 16 unrelated SPG5 families in our cohort. Figure S5. Pedigree, sequencing chromatograms of 5 ARHSP families in our cohort. (DOCX $9157 \mathrm{~kb}$ )

\section{Abbreviations}

AAA: ATPases associated with diverse cellular activities; AAO: Age at onset; AD: Autosomal dominant; ADHSP: Autosomal dominant hereditary spastic paraplegia; AR: Autosomal recessive; ARHSP: Autosomal recessive hereditary spastic paraplegia; DCFH-DA: Dichlorofluorescein diacetate;

DMEM: Dulbecco's modified Eagle's medium; ExAC: Exome Aggregation Consortium database; FBS: Fetal bovine serum; HSP: Hereditary spastic paraplegias; ICH: Immunocytochemistry; LOF: Loss-of-function; LPOs: LAMP1positive organelles; MIT: Microtubule interacting and trafficking; MLPA: Multiplex ligation-dependent probe amplification; MMP: Mitochondrial membrane potential; MRI: Magnetic resonance images; MTBD: Microtubulebinding domain; NGS: Next generation sequencing; PCR: Polymerase chain reaction; qPCR: Quantification PCR; ROS: Reactive oxygen species; RTPCR: Reverse transcription polymerase chain reaction; SPG: Spastic paraplegia; TM: Transmembrane; VDAC: Voltage-dependent anion channel

\section{Acknowledgements}

We sincerely thank the patients and their relatives for participation.

\section{Funding}

This work was supported by the grant 81322017,81771230 and U1505222 from the National Natural Science Foundation of China, grant NCET-13-0736 from Program for New Century Excellent Talents in University, grant 2017XQ1072 from Startup Fund for scientific research of Fujian Medical University, National Key Clinical Specialty Discipline Construction Program and Key Clinical Specialty Discipline Construction Program of Fujian.

\section{Availability of data and materials}

All data generated or analysed during this study are included in this published article [and its supplementary information files].

\section{Authors' contributions}

$X L$ and WJC designed this study. ELD, CW and SW wrote the initial manuscript and constructed the figures. XL, WJC and NW contributed to the editing of the manuscript, figures and tables. ELD and CW performed the Data Analysis. SW, XHL, YQL and HZS performed the sample and history collection. $\mathrm{XL}, \mathrm{ELD}, \mathrm{MZ}, \mathrm{JH}$ and $\mathrm{LXM}$ performed the mutation functional study in vitro. All authors read and approved the final manuscript.

\section{Ethics approval and consent to participate}

Informed written consents were obtained from all the participants. This study was approved by the Ethics Committee of the First Affiliated Hospital of Fujian Medical University (FYYY2006-01-19-01).

\section{Consent for publication}

Not applicable.

\section{Competing interests}

The authors declare that they have no competing interests.

\section{Publisher's Note}

Springer Nature remains neutral with regard to jurisdictional claims in published maps and institutional affiliations.

\section{Author details}

${ }^{1}$ Department of Neurology and Institute of Neurology, The First Affiliated Hospital of Fujian Medical University, Fuzhou 350005, China. ${ }^{2}$ Department of Anatomy, Histology and Embryology, Shanghai Medical College, Fudan University, Shanghai 200032, China. ${ }^{3}$ Fujian Key Laboratory of Molecular Neurology, Fujian Medical University, Fuzhou 350005, China.

Received: 13 November 2017 Accepted: 26 June 2018

Published online: 06 July 2018

\section{References}

1. Morais S, Raymond L, Mairey M, Coutinho P, Brandão E, Ribeiro P, Loureiro $J$, Sequeiros J, Brice A, Alonso I, et al. Massive sequencing of 70 genes reveals a myriad of missing genes or mechanisms to be uncovered in hereditary spastic paraplegias. Eur J Hum Genet. 2017;25:1217-28.

2. Ruano L, Melo C, Silva MC, Coutinho P. The global epidemiology of hereditary ataxia and spastic paraplegia: a systematic review of prevalence studies. Neuroepidemiology. 2014;42:174-83.

3. Lo Giudice T, Lombardi F, Santorelli FM, Kawarai T, Orlacchio A. Hereditary spastic paraplegia: clinical-genetic characteristics and evolving molecular mechanisms. Exp Neurol. 2014;261:518-39.

4. Salinas S, Proukakis C, Crosby A, Warner $\Pi$. Hereditary spastic paraplegia: clinical features and pathogenetic mechanisms. Lancet Neurol. 2008;7:1127-38.

5. de Souza PV, de Rezende Pinto WB, de Rezende Batistella GN, Bortholin T, Oliveira AS. Hereditary spastic paraplegia: clinical and genetic hallmarks. Cerebellum. 2017;16:525-51.

6. Tesson C, Koht J, Stevanin G. Delving into the complexity of hereditary spastic paraplegias: how unexpected phenotypes and inheritance modes are revolutionizing their nosology. Hum Genet. 2015:134:511-38.

7. Estrada-Cuzcano A, Martin S, Chamova T, Synofzik M, Timmann D, Holemans T, Andreeva A, Reichbauer J, De Rycke R, Chang DI, et al. Loss-of-function mutations in the ATP13A2/PARK9 gene cause complicated hereditary spastic paraplegia (SPG78). Brain. 2017;140:287-305.

8. Loureiro JL, Miller-Fleming L, Thieleke-Matos C, Magalhaes P, Cruz VT, Coutinho P, Sequeiros J, Silveira I. Novel SPG3A and SPG4 mutations in dominant spastic paraplegia families. Acta Neurol Scand. 2009;119: 113-8.

9. Schüle R, Wiethoff S, Martus P, Karle KN, Otto S, Klebe S, Klimpe S, Gallenmüller C, Kurzwelly D, Henkel D, et al. Hereditary spastic paraplegia: Clinicogenetic lessons from 608 patients. Ann Neurol. 2016:79:646-58.

10. Kara E, Tucci A, Manzoni C, Lynch DS, Elpidorou M, Bettencourt C, Chelban V, Manole A, Hamed SA, Haridy NA, et al. Genetic and phenotypic characterization of complex hereditary spastic paraplegia. Brain. 2016:139:1904-18.

11. Fink JK. Hereditary spastic paraplegia: clinico-pathologic features and emerging molecular mechanisms. Acta Neuropathol. 2013;126:307-28.

12. Solowska JM, Baas PW. Hereditary spastic paraplegia SPG4: what is known and not known about the disease. Brain. 2015;138:2471-84.

13. Harding AE. Classification of the hereditary ataxias and paraplegias. Lancet. 1983;1:1151-5.

14. McDermott CJ, Grierson AJ, Wood JD, Bingley M, Wharton SB, Bushby KM Shaw PJ. Hereditary spastic paraparesis. Disrupted intracellular transport associated with spastin mutation. Ann Neurol. 2003;54:748-59.

15. Hazan J, Fonknechten N, Mavel D, Paternotte C, Samson D, Artiguenave F, Davoine CS, Cruaud C, Durr A, Wincker P, et al. Spastin, a new AAA protein, is altered in the most frequent form of autosomal dominant spastic paraplegia. Nat Genet. 1999;23:296-303

16. Shoukier M, Neesen J, Sauter SM, Argyriou L, Doerwald N, Pantakani DV, Mannan AU. Expansion of mutation spectrum, determination of mutation cluster regions and predictivestructural classification of SPAST mutations in hereditary spastic paraplegia. Eur J Hum Genet. 2009;17:187-94.

17. Richards S, Aziz N, Bale S, Bick D, Das S, Gastier-Foster J, Grody WW, Hegde M, Lyon E, Spector E, et al. Standards and guidelines for the interpretation 
of sequence variants: a joint consensus recommendation of the American College of Medical Genetics and Genomics and theAssociation for molecular pathology. Genet Med. 2015;17:405-24.

18. Stevanin G, Santorelli FM, Azzedine H, Coutinho P, Chomilier J, Denora PS, Martin E, Ouvrard-Hernandez AM, Tessa A, Bouslam N, et al. Mutations in SPG11, encoding spatacsin, are a major cause of spastic paraplegia with thin corpus callosum. Nat Genet. 2007;39:366-72.

19. Tsaousidou MK, Ouahchi K, Warner TT, Yang Y, Simpson MA, Laing NG, Wilkinson PA, Madrid RE, Patel H, Hentati F, et al. Sequence Alterations within CYP7B1 Implicate Defective Cholesterol Homeostasis in MotorNeuron Degeneration. Am J Hum Genet. 2008;82:510-5.

20. Ueki I, Kimura A, Nishiyori A, Chen HL, Takei H, Nittono H, Kurosawa T. Neonatal cholestatic liver disease in an Asian patient with a homozygous mutation in the oxysterol 7alpha-hydroxylase gene. J Pediatr Gastroenterol Nutr. 2008:46:465-9.

21. Havlicek S, Kohl Z, Mishra HK, Prots I, Eberhardt E, Denguir N, Wend H, Plötz S, Boyer L, Marchetto MC, et al. Gene dosage-dependent rescue of HSP neurite defects in SPG4 patients' neurons. Hum Mol Genet. 2014;23:2527-41.

22. Okamoto K, Shaw JM. Mitochondrial morphology and dynamics in yeast and multicellular eukaryotes. Annu Rev Genet. 2005;39:503-36.

23. Shoshan-Barmatz V, Krelin Y, Chen Q. VDAC1 as a player in mitochondriamediated apoptosis and target for modulating apoptosis. Curr Med Chem. 2017;24:4435-46.

24. Arif T, Krelin Y, Shoshan-Barmatz V. Reducing VDAC1 expression induces a non-apoptotic role for pro-apoptotic proteins in cancer cell differentiation. Biochim Biophys Acta. 1857;2016:1228-42.

25. Connolly NMC, Theurey P, Adam-Vizi V, Bazan NG, Bernardi P, Bolaños JP, Culmsee C, Dawson VL, Deshmukh M, Duchen MR, et al. Guidelines on experimental methods to assess mitochondrial dysfunction in cellular models of neurodegenerative diseases. Cell Death Differ. 2018;25:542-72.

26. Chang J, Lee S, Blackstone C. Spastic paraplegia proteins spastizin and spatacsin mediate autophagic lysosome reformation. J Clin Invest. 2014; 124:5249-62.

27. Klionsky DJ, Abdelmohsen K, Abe A, Abedin MJ, Abeliovich H, Acevedo Arozena A, Adachi H, Adams CM, Adams PD, Adeli K, et al. Guidelines for the use and interpretation of assays for monitoring autophagy (3rd edition). Autophagy. 2016;12:1-222.

28. Meijer IA, Hand CK, Cossette P, Figlewicz DA, Rouleau GA. Spectrum of SPG4 mutations in a large collection of north American families with hereditary spastic paraplegia. Arch Neurol. 2002;59:281-6.

29. Blair MA, Riddle ME, Wells JF, Breviu BA, Hedera P. Infantile onset of hereditary spastic paraplegia poorly predicts the genotype. Pediatr Neurol. 2007;36:382-6

30. Orlacchio A, Montieri P, Babalini C, Gaudiello F, Bernardi G, Kawarai T. Lateonset hereditary spastic paraplegia with thin corpus callosum caused by a new SPG3A mutation. J Neurol. 2011;258:1361-3.

31. Goizet C, Boukhris A, Mundwiller E, Tallaksen C, Forlani S, Toutain A, Carriere N, Paquis V, Depienne C, Durr A, et al. Complicated forms of autosomal dominant hereditary spastic paraplegia are frequent in SPG10. Hum Mutat. 2009;30:E376-85.

32. Muglia M, Citrigno L, D'Errico E, Magariello A, Distaso E, Gasparro AA, Scarafino A, Patitucci A, Conforti FL, Mazzei R, et al. A novel KIF5A mutation in an Italian family marked by spastic paraparesis and congenital deafness. J Neurol Sci. 2014;343:218-20.

33. Beetz C, Nygren AO, Schickel J, Auer-Grumbach M, Burk K, Heide G, Kassubek J, Klimpe S, Klopstock T, Kreuz F, et al. High frequency of partial SPAST deletions in autosomal dominant hereditary spastic paraplegia. Neurology. 2006;67:1926-30.

34. Svenson IK, Ashley-Koch AE, Gaskell PC, Riney TJ, Cumming WJ, Kingston HM, Hogan EL, Boustany RM, Vance JM, Nance MA, et al. Identification and expression analysis of spastin gene mutations in hereditary spastic paraplegia. Am J Hum Genet. 2001;68:1077-85

35. Solowska JM, Morfini G, Falnikar A, Himes BT, Brady ST, Huang D, Baas PW. Quantitative and functional analyses of spastin in the nervous system: implications for hereditary spastic paraplegia. J Neurosci. 2008; 28:2147-57

36. Tortosa E, Adolfs Y, Fukata M, Pasterkamp RJ, Kapitein LC, Hoogenraad CC. Dynamic Palmitoylation targets MAP6 to the axon to promote microtubule stabilization during neuronal polarization. Neuron. 2017;94(4):809-25.

37. Baas PW, Karabay A, Qiang L. Microtubules cut and run. Trends Cell Biol. 2005;15:518-24
38. Ahmad FJ, He Y, Myers KA, Hasaka TP, Francis F, Black MM, Baas PW. Effects of dynactin disruption and dynein depletion on axonal microtubules. Traffic. 2006;7:524-37.

39. Fang C, Bourdette D, Banker G. Oxidative stress inhibits axonal transport: implications for neurodegenerative diseases. Mol Neurodegener. 2012;7:29.

40. Abrahamsen G, Fan Y, Matigian N, Wali G, Bellette B, Sutharsan R, Raju J, Wood SA, Veivers D, Sue CM, et al. A patient-derived stem cell model of hereditary spastic paraplegia with SPASTmutations. Dis Model Mech. 2013;6:489-502.

41. Denton KR, Lei L, Grenier J, Rodionov V, Blackstone C, Li XJ. Loss of spastin function results in disease-specific axonal defects in human pluripotent stem cell-based models of hereditary spastic paraplegia. Stem Cells. 2014;32:414-23.

42. Wharton SB, McDermott CJ, Grierson AJ, Wood JD, Gelsthorpe C, Ince PG, Shaw PJ. The cellular and molecular pathology of the motor system in hereditary spastic paraparesis due to mutation of the spastin gene. J Neuropathol Exp Neurol. 2003;62:1166-77.

43. Paisan-Ruiz C, Nath P, Wood NW, Singleton A, Houlden H. Clinical heterogeneity and genotype-phenotype correlations in hereditary spastic paraplegia because of Spatacsin mutations (SPG11). Eur J Neurol. 2008;15:1065-70.

44. Samaranch L, Riverol M, Masdeu JC, Lorenzo E, Vidal-Taboada JM, Irigoyen J, Pastor MA, de Castro P, Pastor P. SPG11 compound mutations in spastic paraparesis with thin corpus callosum. Neurology. 2008;71(5):332-6.

45. Choquet K, Tetreault M, Yang S, La Piana R, Dicaire MJ, Vanstone MR, Mathieu J, Bouchard JP, Rioux MF, Rouleau GA, et al. SPG7 mutations explain a significant proportion of French Canadian spastic ataxia cases. Eur J Hum Genet. 2016;24:1016-21.

46. Klebe S, Depienne C, Gerber S, Challe G, Anheim M, Charles P, Fedirko E, Lejeune E, Cottineau J, Brusco A, et al. Spastic paraplegia gene 7 in patients with spasticity and/or optic neuropathy. Brain. 2012;135:2980-93.

47. Goizet C, Boukhris A, Durr A, Beetz C, Truchetto J, Tesson C, Tsaousidou M, Forlani S, Guyant-Maréchal L, Fontaine B, et al. CYP7B1 mutations in pure and complex forms of hereditary spastic paraplegia type 5. Brain. 2009;132: 1589-600.

48. R S, Brandt E, Karle KN, Tsaousidou M, Klebe S, Klimpe S, Auer-Grumbach M, Crosby AH, Hübner CA, Schöls L, et al. Analysis of CYP7B1 in nonconsanguineous cases of hereditary spastic paraplegia. Neurogenetics. 2009; 10:97-104.

49. Stevanin G, Azzedine $H$, Denora P, Boukhris A, Tazir M, Lossos A, Rosa AL, Lerer I, Hamri A, Alegria $P$, et al. Mutations in SPG11 are frequent in autosomal recessive spastic paraplegia with thin corpus callosum, cognitive decline and lower motor neuron degeneration. Brain. 2008;131:772-84.

50. Renvoise B, Chang J, Singh R, Yonekawa S, FitzGibbon EJ, Mankodi A, Vanderver A, Schindler A, Toro C, Gahl WA, et al. Lysosomal abnormalities in hereditary spastic paraplegia types SPG15 and SPG11. Ann Clin Transl Neurol. 2014;1:379-89.

51. Vantaggiato C, Crimella C, Airoldi G, Polishchuk R, Bonato S, Brighina E, Scarlato M, Musumeci O, Toscano A, Martinuzzi A, et al. Defective autophagy in spastizin mutated patients with hereditary spastic paraparesis type 15. Brain. 2013;136:3119-39.

52. Mishra HK, Prots I, Havlicek S, Kohl Z, Perez-Branguli F, Boerstler T, Anneser L, Minakaki G, Wend H, Hampl M, et al. GSK3ß-dependent dysregulation of neurodevelopment in SPG11-patient iPSC model. Ann Neurol. 2016; https:// doi.org/10.1002/ ana.24633.

53. Pérez-Brangulí F, Mishra HK, Prots I, Havlicek S, Kohl Z, Saul D, Rummel C, Dorca-Arevalo J, Regensburger M, Graef D, et al. Dysfunction of spatacsin leads to axonal pathology in SPG11-linked hereditary spastic paraplegia. Hum Mol Genet. 2014:23:4859-74.

54. Varga RE, Khundadze M, Damme M, Nietzsche $S$, Hoffmann B, Stauber T, Koch N, Hennings JC, Franzka P, Huebner AK, et al. In vivo evidence for lysosome depletion and impaired Autophagic clearance in hereditary spastic paraplegia type SPG11. PLoS Genet. 2015;11:e1005454.

55. Walker C, El-Khamisy SF. Perturbed autophagy and DNA repair converge to promote neurodegeneration in amyotrophic lateral sclerosis and dementia. Brain. 2018; https://doi.org/10.1093/brain/awy076. 